\title{
Real-World Efficacy of Letermovir Prophylaxis for Cytomegalovirus Infection After Allogeneic Hematopoietic Stem Cell Transplantation: A Single- Center Retrospective Analysis
}

Hideaki Yoshimura

Kansai Medical University

Atsushi Satake ( $\square$ satake_at@yahoo.co.jp )

Kansai Medical University

Yuka Ishii

Kansai Medical University

Jun Ichikawa

Kansai Medical University

Ryo Saito

Kansai Medical University

Akiko Konishi

Kansai Medical University

Masaaki Hotta

Kansai Medical University

Takahisa Nakanishi

Kansai Medical University

Shinya Fujita

Kansai Medical University

Tomoki lto

Kansai Medical University

Kazuyoshi Ishii

Kansai Medical University

Shosaku Nomura

Kansai Medical University

\section{Research Article}

Keywords: Real-world, letermovir, cytomegalovirus, infection, hematopoietic

Posted Date: November 10th, 2021 
DOI: https://doi.org/10.21203/rs.3.rs-1029207/v1

License: (c) (1) This work is licensed under a Creative Commons Attribution 4.0 International License. Read Full License 


\section{Abstract}

Cytomegalovirus (CMV) infection is a common complication following allogeneic hematopoietic stem cell transplantation (aHSCT) and is associated with increased mortality. Letermovir (LET) is a novel antiviral drug used to prevent CMV infection. We retrospectively analyzed 111 consecutive patients who underwent aHSCT to evaluate the efficacy of LET prophylaxis for clinically significant CMV (cSCMV) infection in real-world situations and analyzed the influence of LET on transplant outcomes. Thirty-eight patients who were prophylactically administered LET were compared with 73 patients without LET prophylaxis for aHSCT. On Day 180, the cumulative incidence of csCMV infection in patients who received LET prophylaxis was significantly lower than that in patients without LET prophylaxis $(29.7 \%$ vs. $56.2 \%, \mathrm{P}<0.001)$. Among patients who developed csCMV infection, the interval from aHSCT to the initiation of preemptive therapy was significantly longer in patients who received LET prophylaxis than in those who did not receive LET prophylaxis ( 129.5 days vs. 42 days, $\mathrm{P}<0.001)$. Six-month overall survival was $86.1 \%$ in patients who received LET prophylaxis and $66.8 \%$ in the non-LET group $(P=0.035)$. LET prophylaxis was highly effective in preventing csCMV infection in real-world situations and could potentially improve transplant outcomes, particularly when initiated early after transplantation.

\section{Introduction}

Cytomegalovirus (CMV) infection is a major infectious complication of allogeneic hematopoietic stem cell transplantation (aHSCT). A considerable number of CMV infections can be prevented by preemptive antiviral therapy along with monitoring for CMV reactivation. However, even in patients receiving preemptive therapy, CMV reactivation remains a major risk factor for non-relapse mortality (NRM) after aHSCT. In addition to the direct effects of CMV infection, the virus can indirectly influence mortality ${ }^{1-4}$ through clinical events associated with virus seropositivity or CMV reactivation but not with the viral disease itself ${ }^{2,5-8}$. Following aHSCT, CMV infection is associated with an increased risk of secondary bacterial and fungal infections, increased NRM, as well as a potentially increased risk of graft-versus-host disease (GVHD).

In this context, the prevention of CMV reactivation after aHSCT is of vital importance. However, there are no well-tolerated antiviral agents that can be safely prescribed prophylactically after aHSCT. Antiviral agents such as ganciclovir, foscarnet, cidofovir, and valganciclovir are effective against CMV infection. However, due to their inherent toxicities (particularly myelotoxicity and nephrotoxicity), pre-emptive therapy along with continuous monitoring remains the standard of care for CMV infection following aHSCT.

Letermovir (LET), an antiviral agent that inhibits CMV replication by binding to the components of the terminase complex (UL51, UL56, or both), was recently approved in Japan (May 2018) for prophylaxis against CMV infections in aHSCT recipients ${ }^{9,10}$. The efficacy and safety of a 14-week LET regimen in CMV-seropositive HSCT recipients have been validated in a randomized phase 3 study ${ }^{10}$. The incidence of clinically significant CMV (csCMV) infection at 24 weeks was significantly lower in patients receiving 
LET versus placebo ( $37.5 \%$ vs. $60.6 \%$, respectively, $\mathrm{P}<0.001$ ), with no major toxic effects, leading to the approval of LET for primary CMV prophylaxis in aHSCT recipients in Japan in March 2018. Prophylactic LET treatment after aHSCT has also been recommended in the 2017 European Conference on Infections in Leukemia (ECIL 7) guidelines ${ }^{11}$. Although NRM among LET-treated recipients was not significantly lower than that among placebo recipients in the phase 3 study, the prophylactic use of LET may reduce NRM by preventing CMV-induced indirect effects and could thereby improve transplant outcomes. Nevertheless, there is limited real-world data on the prophylactic effect of LET, and the impact of LET prophylaxis on NRM has not been fully evaluated.

We initiated CMV prophylaxis with LET as the standard of care at our institution since LET became commercially available. In this retrospective study, we investigated whether the positive effects of LET for csCMV infection in the phase 3 study would be confirmed and evaluated the influence of LET prophylaxis on transplant outcomes in real-world situations.

\section{Results}

\section{Patient characteristics}

A total of 111 patients were included in the study. Seventy-three patients underwent aHSCT before LET approval in Japan (January 2011-April 2018), and 38 patients underwent the procedure after LET approval (March 2018-May 2020). All patients who underwent aHSCT after LET approval were prophylactically administered LET against csCMV infection from the day of transplantation. The clinical and transplant characteristics of all patients are presented in Table 1. Among the 38 patients who received LET, 35 (92.1\%) were CMV-seropositive, and 3 (7.9\%) were CMV seronegative but received aHSCT from CMV-seropositive donors. These two groups of patients exhibited no significant differences, except for CMV serostatus and stem cell source. Regarding CMV serostatus, the proportion of seropositive recipients was comparable between patients with and without LET prophylaxis $(92.1 \% \mathrm{vs}$. $90.4 \%$ ). Additionally, peripheral blood stem cell transplantation was preferentially used in patients receiving LET prophylaxis. The risk of csCMV infection was $73.7 \%$ in patients with LET and $71.2 \%$ in patients without LET prophylaxis $(P=0.827)$. 
Table 1

Baseline patient characteristics at transplantation

\begin{tabular}{|c|c|c|c|}
\hline Characteristic, n (\%) & Letermovir $(-)(n=73)$ & Letermovir $(+)(n=38)$ & $P$-value \\
\hline Age, years & $48(17-70)$ & $49(18-68)$ & 0.871 \\
\hline \multicolumn{4}{|l|}{ Median (Range) } \\
\hline Sex & $42(57.5)$ & $25(65.8)$ & \multirow[t]{3}{*}{0.422} \\
\hline Male & $31(42.5)$ & $13(34.2)$ & \\
\hline \multicolumn{3}{|l|}{ Female } & \\
\hline CMV serostatus & $4(5.5)$ & $0(0.0)$ & \multirow[t]{5}{*}{0.082} \\
\hline D-/R- & $38(52.1)$ & $13(34.2)$ & \\
\hline $\mathrm{D}-/ \mathrm{R}+$ & $3(4.1)$ & $3(7.9)$ & \\
\hline$D+/ R-$ & $28(38.4)$ & $22(57.9)$ & \\
\hline \multicolumn{3}{|l|}{$\mathrm{D}+/ \mathrm{R}+$} & \\
\hline Disease & $24(32.9)$ & $13(34.2)$ & \multirow[t]{9}{*}{0.320} \\
\hline Acute lymphoblastic leukemia & $24(32.9)$ & $15(39.5)$ & \\
\hline Acute myeloid leukemia & $9(12.3)$ & $4(10.5)$ & \\
\hline Myelodysplastic syndrome & $2(2.7)$ & $2(5.3)$ & \\
\hline Non-Hodgkin's lymphoma & $7(9.6)$ & $0(0.0)$ & \\
\hline Adult T cell leukemia/lymphoma & $2(2.7)$ & $1(2.6)$ & \\
\hline Chronic myeloid leukemia & $2(2.7)$ & $2(5.3)$ & \\
\hline Plasma cell leukemia & $3(4.1)$ & $1(2.6)$ & \\
\hline \multicolumn{3}{|l|}{ Other } & \\
\hline DRI at HSCT & $41(56.2)$ & $22(57.9)$ & \multirow[t]{3}{*}{1.000} \\
\hline Low / Intermediate & $32(43.8)$ & $16(42.1)$ & \\
\hline High / Very high & & & \\
\hline
\end{tabular}

Abbreviations: aHSCT, allogeneic hematopoietic stem cell transplantation; CMV, cytomegalovirus; $D$, donor; DRI, disease risk index; GVHD, graft-versus-host disease; HCT-Cl, hematopoietic cell transplantation-comorbidity index; HLA, human leukocyte antigen; HSCT, hematopoietic stem cell transplantation; MAC, myeloablative conditioning; PTCY, posttransplant cyclophosphamide; R, recipient; RIC, reduced-intensity conditioning 


\begin{tabular}{|c|c|c|c|}
\hline Characteristic, n (\%) & Letermovir $(-)(n=73)$ & Letermovir $(+)(n=38)$ & $P$-value \\
\hline HLA matching and donor type & $9(12.3)$ & $5(13.2)$ & 0.526 \\
\hline Matched related & $28(38.4)$ & $18(47.4)$ & \\
\hline Matched unrelated & $25(34.2)$ & $8(21.1)$ & \\
\hline Mismatched unrelated & $11(15.1)$ & $7(18.4)$ & \\
\hline \multicolumn{4}{|l|}{ Haploidentical related } \\
\hline $\mathrm{HCT}-\mathrm{Cl}$ & $61(83.6)$ & $36(94.7)$ & 0.133 \\
\hline$<2$ & $12(16.4)$ & $2(5.3)$ & \\
\hline \multicolumn{4}{|l|}{$\geq 2$} \\
\hline Stem cell source & $38(52.1)$ & $14(36.8)$ & 0.031 \\
\hline Bone marrow & $20(27.4)$ & $20(52.6)$ & \\
\hline Peripheral blood & $15(20.5)$ & $4(10.5)$ & \\
\hline \multicolumn{4}{|l|}{ Cord blood } \\
\hline Conditioning regimen & $51(69.9)$ & $22(57.9)$ & 0.215 \\
\hline MAC & $22(30.1)$ & $16(42.1)$ & \\
\hline \multicolumn{4}{|l|}{ RIC } \\
\hline GVHD prophylaxis & $15(20.5)$ & $7(18.4)$ & 0.424 \\
\hline Cyclosporine-based & $51(69.9)$ & $24(63.2)$ & \\
\hline Tacrolimus-based & $7(9.6)$ & $7(18.4)$ & \\
\hline \multicolumn{4}{|l|}{ Tacrolimus-based/PTCY } \\
\hline Antithymocyte globulin use & $5(6.8)$ & $2(5.3)$ & 1.000 \\
\hline \multicolumn{4}{|l|}{ Yes } \\
\hline Prior aHSCT & $10(13.7)$ & 5 (13.2) & 1.000 \\
\hline Yes & & & \\
\hline
\end{tabular}

Abbreviations: aHSCT, allogeneic hematopoietic stem cell transplantation; CMV, cytomegalovirus; $D$, donor; DRI, disease risk index; GVHD, graft-versus-host disease; HCT-Cl, hematopoietic cell transplantation-comorbidity index; HLA, human leukocyte antigen; HSCT, hematopoietic stem cell transplantation; MAC, myeloablative conditioning; PTCY, posttransplant cyclophosphamide; R, recipient; RIC, reduced-intensity conditioning 


\begin{tabular}{|llll|}
\hline Characteristic, $\mathbf{n}(\%)$ & Letermovir $(-)(\mathbf{n = 7 3 )}$ & Letermovir $(+)(\mathbf{n = 3 8 )}$ & $P$-value \\
\hline Risk of CMV disease & $52(71.2)$ & $28(73.7)$ & 0.827 \\
High risk & $21(28.8)$ & $10(26.3)$ & \\
Low risk & & & \\
\hline $\begin{array}{l}\text { Abbreviations: aHSCT, allogeneic hematopoietic stem cell transplantation; CMV, cytomegalovirus; D, } \\
\text { donor; DRI, disease risk index; GVHD, graft-versus-host disease; HCT-Cl, hematopoietic cell } \\
\text { transplantation-comorbidity index; HLA, human leukocyte antigen; HSCT, hematopoietic stem cell } \\
\text { transplantation; MAC, myeloablative conditioning; PTCY, posttransplant cyclophosphamide; R, } \\
\text { recipient; RIC, reduced-intensity conditioning }\end{array}$ & \\
\hline
\end{tabular}

\section{Efficacy of LET for clinically significant CMV infection}

The cumulative incidence of csCMV infection by day 180 after aHSCT was significantly lower among patients receiving LET prophylaxis than among those without LET prophylaxis (29.7\% [95\% confidence interval (Cl): 15.8-44.9] vs. 56.2\% [95\% Cl: 43.9-66.7]; $P<0.001$ ) (Fig. 1a). The median time from aHSCT to the development of $\mathrm{csCMV}$ infection was significantly longer in patients who received LET prophylaxis compared to that of those without LET prophylaxis (129.5 [range: $26-150$ ] days vs. 42 [range: 18-87] days; $\mathrm{P}<0.001$ ) (Fig. 2a, b). One patient (2.6\%) with LET prophylaxis developed CMV colitis, whereas 5 patients (6.8\%) without LET prophylaxis developed CMV disease (colitis: 3 , pneumonia: 2, retinitis: 1 , hepatitis: 1). The median number of antigenemia-positive cells at the time of initiation of anti-CMV therapy in patients receiving LET prophylaxis $(6.5 / 50,000$ WBCs [range: $2-33 / 50,000$ WBCs]) was comparable to that in patients without LET prophylaxis $(5 / 50,000 \mathrm{WBC}$ [range: $1-229 / 50,000 \mathrm{WBC}$ ] $)(\mathrm{P}$ $=0.564$ ) (Fig. 2a). The median duration of treatment for the initial csCMV infection was 15 (range: 4-57) days in patients with LET prophylaxis and 20 days (range: 4-68 days) in patients without LET prophylaxis $(P=0.483)$ (Fig. 2c). Relapse of csCMV infection after initial treatment for csCMV infection developed in $8 / 12(66.7 \%)$ patients treated with LET and in of 29/41 (70.1\%) patients not treated with LET (data not shown). LET prophylaxis was more effective in patients at high risk for CMV infection (37.0\% [95\% Cl: $19.0-55.0$ ] vs. $61.5 \%$ [95\% Cl: $46.7-73.4$ ] in patients without LET prophylaxis, $\mathrm{P}=0.003$ ) than in patients with low risk $(10.0 \%$ [95\% Cl: $5.0-37.4]$ in patients treated with LET vs. $42.9 \%$ [95\% Cl: 21.3-62.9] in patients without LET prophylaxis, $P=0.096$ ) (Fig. 1b, c). The prophylactic effect of LET for csCMV infection by day 180 following aHSCT was confirmed in older patients ( $\geq 55$ years) $(18.2 \%$ [ $95 \%$ Cl: 2.3-83.1] in patients with LET prophylaxis vs. $68.0 \%$ [95\% Cl: 44.8-83.1] in patients without LET prophylaxis, $P=0.009$ ) (Fig. 1d).

\section{Outcomes of aHSCT}

To evaluate the effects of LET prophylaxis on transplant outcomes, the incidence of NRM, disease mortality, and overall survival (OS) at 180 days and 1 year after aHSCT were examined among the subsets of patients with or without LET prophylaxis. The cumulative incidence of NRM at 180 days after aHSCT in patients with LET prophylaxis was lower, but without statistical significance, compared to that in patients without LET prophylaxis (11.0\% [95\% Cl: 3.4-23.7] vs. $22.1 \%$ [95\% Cl: $13.3-32.2], \mathrm{P}=0.148)$ 
(Fig. 3a). In addition, the cumulative incidence of disease mortality at 180 days was lower, but not significantly, compared to that in patients without LET prophylaxis $(2.9 \%$ [95\% Cl: $2.0-13.0]$ vs. $11.1 \%$ [95\% Cl: 5.2-19.7], $\mathrm{P}=0.146$ ) (Fig. 3b). OS at 180 days after aHSCT in patients with LET prophylaxis was significantly higher than that in patients without LET prophylaxis ( $86.1 \%$ [95\% Cl: $69.8-94.0]$ vs. $66.8 \%$ [95\% Cl: 54.7-76.4], $\mathrm{P}=0.035$ ) (Fig. 3a). The cumulative incidence of NRM at 1 year after aHSCT in patients with LET prophylaxis was marginally lower than that in patients without LET prophylaxis $(14.1 \%$ [95\% Cl: 5.0-27.7] vs. 30.5\% [95\% Cl: 20.2-41.4], P = 0.067) (Fig. 3a), whereas the cumulative incidence of disease mortality at 1 year after aHSCT was comparable between the two groups $(14.8 \%$ [95\% Cl: $5.3-$ 28.9 ] vs. $13.9 \%$ [95\% Cl: $7.1-23.0], P=0.981$ ) (Fig. 3b). Finally, OS at 1 year after aHSCT in patients with LET prophylaxis was not significantly higher than that in patients without LET prophylaxis $(67.9 \%$ [95\% Cl: 49.4-80.8] vs. $55.6 \%$ [95\% Cl: 43.4-66.2], P = 0.147) (Fig. 3c).

Univariate analysis revealed that three factors were significantly associated with OS at 180 days postaHSCT (Table 2): hematopoietic cell transplantation-specific comorbidity index ( $\mathrm{HCT}-\mathrm{Cl})(\geq 2)(\mathrm{P}<0.01)$, refined disease risk index $(D R I)$ at transplantation $(P<0.001)$, and prophylaxis with LET $(P<0.05)$. Multivariate analysis revealed that HCT-Cl (hazard ratio: 2.34 [95\% Cl: 1.20-5.36]) and DRI at transplantation (4.10 [95\% Cl: 1.81-9.31]) were unfavorable risk factors for 180-day OS. 
Table 2

Univariate and multivariate analysis for 180-day overall survival

\begin{tabular}{|c|c|c|c|c|c|c|}
\hline \multirow[t]{2}{*}{ Variable } & \multicolumn{3}{|c|}{ Univariate analysis } & \multicolumn{3}{|c|}{ Multivariate analysis } \\
\hline & HR & $95 \% \mathrm{Cl}$ & $\mathrm{p}$ value & HR & $95 \% \mathrm{Cl}$ & $\mathrm{p}$ value \\
\hline Age: $<55$ vs. $\geq 55$ years & 1.78 & $0.86-3.71$ & 0.121 & - & - & - \\
\hline HCT-Cl: $<2$ vs. $\geq 2$ & 3.20 & $1.42-7.24$ & 0.005 & 2.34 & $1.02-5.36$ & 0.044 \\
\hline Conditioning: MAC vs. RIC & 1.37 & $0.65-2.87$ & 0.406 & - & - & - \\
\hline $\begin{array}{l}\text { Recipient CMV serostatus: } \\
\text { Negative vs. Positive }\end{array}$ & 1.44 & $0.34-6.06$ & 0.619 & - & - & - \\
\hline CMV risk: Low vs. High & 1.30 & $0.56-3.05$ & 0.542 & - & - & - \\
\hline \multicolumn{6}{|c|}{ Low/Intermediate vs. High/Very high } & $<0.001$ \\
\hline HLA mismatch: No vs. Yes & 1.57 & $0.75-3.26$ & 0.228 & - & - & - \\
\hline \multicolumn{6}{|l|}{ No vs. Yes } & - \\
\hline Letermovir: No vs. Yes & 0.37 & $0.14-0.97$ & 0.043 & 0.40 & $0.15-1.07$ & 0.067 \\
\hline $\begin{array}{l}\text { Abbreviations: } \mathrm{Cl} \text {, Confideno } \\
\text { versus-host disease; HCT-Cl, } \\
\text { leukocyte antigen; HR, Haza } \\
\text { conditioning }\end{array}$ & $\begin{array}{l}\text { MV, cy } \\
\text { tic ce } \\
\text { t, mye }\end{array}$ & $\begin{array}{l}\text { megalovirus } \\
\text { ransplantat } \\
\text { ablative cor }\end{array}$ & $\begin{array}{l}\text { DRI, dise } \\
\text { n-comol } \\
\text { itioning; }\end{array}$ & $\begin{array}{l}\text { e risk } \\
\text { lity in } \\
\text { C, redi }\end{array}$ & $\begin{array}{l}\text { dex; GVHD, } \\
\text { x; HLA, hum } \\
\text { ed-intensity }\end{array}$ & \\
\hline
\end{tabular}

The causes of death are listed in Table 3. By the end of the data collection period, 13 (34.2\%) patients who received LET had died at a median of 186 (range: $59-771$ ) days after aHSCT; seven (18.4\%) patients died due to NRM, and 6 (15.8\%) died due to relapse or progressive disease (PD). In contrast, 42 patients $(57.5 \%)$ who did not receive LET prophylaxis died within a median of 161 (range: $21-1,964$ ) days after aHSCT, with a higher proportion of patients dying due to NRM; the causes of mortality were categorized as NRM $(n=26[35.6 \%])$ and relapse or PD $(n=16[21.9 \%])$. 
Table 3

Causes of mortality

\begin{tabular}{|lll|}
\hline Cause & \multicolumn{2}{l|}{ Letermovir prophylaxis } \\
& No, $\mathbf{n}(\%)$ Yes, $\mathbf{n}$ (\%) \\
\hline Disease progression/relapse & $16(38)$ & $6(46)$ \\
\hline Acute GVHD & $6(14)$ & $3(23)$ \\
\hline Chronic GVHD & $1(2)$ & $0(0)$ \\
\hline Infection & $4(10)$ & $2(15)$ \\
\hline IPS & $3(7)$ & $1(8)$ \\
\hline VOD/SOS & $3(7)$ & $0(0)$ \\
\hline TMA & $3(7)$ & $1(8)$ \\
\hline Other & $6(14)$ & 0 \\
\hline $\begin{array}{l}\text { Abbreviations: GVHD, graft-versus-host disease; IPS, idiopathic pneumonia syndrome; TMA, } \\
\text { thrombotic microangiopathy; VOD/SOS, veno-occlusive disease/sinusoidal obstructive syndrome }\end{array}$ \\
\hline
\end{tabular}

\section{LET-related adverse events}

The median time to neutrophil engraftment was 18 and 17 days in patients with and without LET prophylaxis, respectively $(P=0.953)$ (Fig. 4a). The median time to platelet engraftment was 34 and 28 days in patients with and without LET prophylaxis $(P=0.567)$, respectively (Fig. $4 \mathrm{~b})$. The median time to reticulocyte engraftment was also similar between the two groups ( 25 vs. 24 days, respectively; $P=0.643$ ) (Fig. 4c). These results indicate that LET did not cause myelotoxicity.

Nausea (grade 1-3) was reported by two (5.3\%) patients who received LET prophylaxis. Of the patients receiving LET prophylaxis, Vertigo (grade 1) and liver injury (grade 1) developed in 1 (2.6\%) patient each (data not shown). One patient discontinued LET because of sustained nausea. The median treatment duration of LET was 100 (range: 26-100) days.

\section{Discussion}

This retrospective study evaluated the prophylactic efficacy of LET against CMV reactivation and the transplant outcomes after aHSCT in clinical practice. The findings demonstrated a definite prophylactic anti-CMV effect of LET, along with an excellent safety profile. Although LET prophylaxis did not significantly improve NRM, LET may have improved OS by inhibiting and delaying csCMV infection.

Preemptive anti-CMV treatment, guided by the monitoring of CMV reactivation after aHSCT, has been confirmed to be a successful approach for CMV disease prophylaxis over the last few decades. This strategy was facilitated by the advent of effective anti-CMV agents, such as ganciclovir, foscarnet, and 
valganciclovir, and with the introduction of sensitive CMV monitoring methods. Nevertheless, seropositivity for CMV remains a risk factor for $\mathrm{NRM}^{4,12,13}$, and CMV reactivation early after aHSCT is associated with increased $\mathrm{NRM}^{3,4}$. The contribution of $\mathrm{CMV}$ reactivation to morbidity and mortality after HSCT is considered to be attributable to indirect effects. CMV shows immunomodulatory activity; for example, it can inhibit interferon- $y$ production and its receptor signal and promote the production of IL-10 in infected host cells. Additionally, CMV can prevent antigen presentation by its peptides and NK cell function. Therefore, CMV reactivation can cause complications associated with the host's immune conditions through these modifications.

Regarding the prophylaxis for csCMV infection, the cumulative incidence at 180 days after aHSCT in the present study was slightly higher than that reported in the phase 3 study. However, compared with the phase 3 study, this investigation included a higher percentage of CMV high-risk patients, such as those who received cord blood transplantation (CBT) and HLA-mismatched hematopoietic stem cell transplantation (HSCT). CBT has been reported to be a risk factor for CMV reactivation or disease $\mathrm{e}^{14,15}$, primarily as a result of the immaturity and dysfunction of lymphocytes in the cord blood ${ }^{16,17}$, and HLA mismatch is also thought to contribute to CMV reactivation ${ }^{18,19}$. T cell-replete aHSCT from an HLA haploidentical donor with post-transplant cyclophosphamide (PTCy) has also been associated with a high risk of $\mathrm{CMV}^{20,21}$. A recent report revealed that PTCy increased the risk of CMV infection in CMVseropositive recipients who received aHSCT from HLA-haploidentical/matched sibling donors compared to recipients who received aHSCT from HLA-matched sibling donors with conventional calcineurin inhibitor-based GVHD prophylaxis. This suggests that PTCy itself may increase the risk of CMV infection in CMV-seropositive recipients regardless of HLA disparity ${ }^{22}$. Alternatively, Terao T et al. reported that LET prophylaxis may be associated with an increased risk of chronic GVHD through an early increase in HLA$\mathrm{DR}^{+}$activated T cells in PTCy-haplorecipients who received $\mathrm{LET}^{23}$. The ECIL 7 recommends real-time quantitative PCR (qPCR) methods to guide the initiation of preemptive therapy and to monitor the response ${ }^{11}$. We used the anti-CMV pp65 monoclonal antibody HRP-C7 assay to evaluate CMV reactivation. Kanda et al. reported that $\mathrm{CMV}$ antigenemia and $\mathrm{QPCR}$ were equally appropriate as $\mathrm{CMV}$ monitoring techniques ${ }^{13}$. Nevertheless, they demonstrated that the threshold for $\mathrm{CMV}$ antigenemia positive cells, usually used for CMV high-risk patients in Japan, may be too low. Therefore, for some Japanese patients, we may be initiating preemptive therapy excessively compared to patients monitored by qPCR.

Consistent with the results of the phase 3 study ${ }^{10}$, the onset of csCMV infection was significantly delayed by LET prophylaxis in the present study. Delayed CMV reactivation has been associated with a reduction in transplant-related mortality in the era of preemptive therapy ${ }^{24}$. Therefore, CMV prophylaxis with LET may reduce transplant-related mortality. In addition, CMV viral load is associated with an increased risk of early death ${ }^{24,25}$. According to the results of the phase 3 study, the median CMV DNA level with csCMV infection in the LET group (223 copies/mL [range: 150-27,946 copies/mL]) was lower than that in the control group (1,014 copies/mL [range: $150-106,830$ copies/mL]), although the median CMV DNA level 
without csCMV infection was comparable in both groups ${ }^{10}$. All-cause mortality at week 24 after aHSCT was lower in the LET group than in the non-LET group, whereas the NRM of the LET group was slightly, but not significantly, lower than that in the non-LET group. In particular, the risk of all-cause mortality in patients with csCMV infection is reduced by LET prophylaxis ${ }^{26}$. Although LET prophylaxis was not associated with improved OS with statistical significance in this study, we believe that LET has the potential to improve transplant outcomes. A recent report from the adult T-cell leukemia/lymphoma (ATL) endemic area of Japan, in which the cumulative incidence of csCMV infections was considerably high, probably owing to the higher susceptibility of ATL patients to CMV infections ${ }^{27}$, suggested that LET reduces transplant-related mortality ${ }^{28}$. Therefore, further analysis with a large-scale survey is needed to clarify the effects of LET on transplant outcomes in clinical practice.

The optimal timing for initiation of LET prophylaxis remains unknown. Unlike the phase 3 study, in which LET administration was initiated at a median of 9 days following HSCT, all patients in this study received LET prophylaxis from the day of aHSCT. We believe that early initiation of LET prophylaxis after aHSCT may enhance the benefits for recipients. The phase 3 study was designed to initiate LET prophylaxis by day 28 after aHSCT ${ }^{10}$. Forty-eight patients already had detectable CMV DNA at the time of LET initiation, and the median day to randomization was longer than that of patients with undetectable CMV DNA (15 days vs. 8 days, $P<0.001)$. The Kaplan-Meier event rate of csCMV infection (44.3\%) was higher than that in patients with undetectable CMV DNA $(18.9 \%)^{29}$. Additionally, pre-engraft CMV DNAemia developed in some patients, as reported previously ${ }^{30}$, although we could not evaluate CMV reactivation before engraftment because we used the CMV pp65 antigenemia assay to detect CMV reactivation. In a recent report from a German group ${ }^{31}$, the median starting time for LET prophylaxis was 10 days later than that in the phase 3 study. The cumulative incidence of csCMV infection through week 14 was significantly lower in the LET group than in the control group. However, unlike the phase 3 study and our present study, the median time to $\mathrm{CMV}$ reactivation in patients with csCMV was similar in both groups. These results emphasize the importance of early initiation of LET treatment.

This was a small-scale retrospective study, and the control group comprised patients who had undergone aHSCT before LET was approved (January 2011 to April 2018). However, transplant outcomes are generally improved by other factors as well, including new antibiotics and examination techniques ${ }^{32}$. Consequently, the transplant outcomes of patients who recently underwent aHSCT without LET prophylaxis might be better than the findings obtained in this study.

In conclusion, LET prophylaxis for csCMV infection was beneficial for aHSCT recipients in a real-world situation. However, a large-scale study is required to ascertain the appropriate timing for LET prophylaxis initiation and confirm the influence of LET on transplant outcomes.

\section{Methods}

\section{Patients and study design}


A total of 125 patients with hematologic malignancy underwent aHSCT between January 2011 and May 2020 at the Kansai Medical University Hospital. Fourteen patients who died before engraftment $(n=9)$ or developed graft failure $(n=5)$ were excluded from this study because CMV infection in these patients could not be evaluated using the pp65 antigenemia assay. Therefore, the remaining 111 patients were retrospectively analyzed. Thirty-eight patients who received LET prophylaxis after aHSCT were retrospectively compared with 73 patients who underwent transplantation before the approval of LET in Japan. The primary endpoint was the cumulative incidence of csCMV (defined as CMV disease or CMV reactivation leading to preemptive treatment) at day 180 following aHSCT. Secondary endpoints included disease mortality, NRM, and OS. All data were obtained using electronic chart reviews. The study protocol complied with the Declaration of Helsinki and was approved by the Research Ethics Committee of the Faculty of Medicine, Kansai Medical University. The need for informed consent was waived due to the retrospective nature of the study by the Research Ethics Committee of the Faculty of Medicine, Kansai Medical University.

\section{Definitions}

Conditioning intensity was categorized according to the Working Group definitions ${ }^{33}$. The HCT-Cl was determined as described by Sorror et al. ${ }^{34}$. Disease risk was determined using the DRI ${ }^{35}$. The clinical stage and severity of acute GVHD were defined according to the modified Glucksberg criteria ${ }^{36}$. Neutrophil engraftment was defined as the first of three consecutive days with an absolute neutrophil count of at least $5 \times 10^{8} / \mathrm{L}$, and platelet engraftment was defined as the first day without platelet transfusion with an absolute platelet count of at least $2 \times 10^{10} / \mathrm{L}$. Reticulocyte engraftment was defined as the first day with a reticulocyte ratio of at least $1 \%$.

\section{CMV risk stratification and management}

A high risk of $C M V$ reactivation and $C M V$ disease was defined as the presence of one or more of the previously reported criteria ${ }^{10}$ with minor modifications: having an HLA (HLA-A, B, C, or DRB1) allelemismatched donor; having a haploidentical donor; the use of umbilical cord blood as the stem-cell source; the use of ex vivo T-cell-depleted grafts; and GVHD $\geq$ grade 2 leading to the use of $\geq 0.5 \mathrm{mg}$ prednisone (or its equivalent) per kilogram of body weight per day.

Patients underwent surveillance for CMV antigenemia at least once weekly from the time of engraftment. Surveillance was performed by the anti-CMV pp65 monoclonal antibody HRP-C7 assay because the polymerase chain reaction (PCR) method to detect CMV reactivation was not yet approved in Japan. CMV reactivation was defined by the initiation of $C M V$ preemptive therapy, which was generally initiated when at least two CMV pp65 antigen-positive cells per 50,000 white blood cells were detected ${ }^{3}$. Anti-CMV drugs (intravenous ganciclovir, foscarnet, or oral valganciclovir) for preemptive treatment were selected at the physician's discretion. CMV disease was diagnosed based on published recommendations ${ }^{37}$ histological examination of a biopsy specimen with positive results of CMV antigenemia or PCR of focal samples. 


\section{Letermovir prophylaxis}

LET prophylaxis was administered from May 2018 upon approval in Japan. At our institution, LET was administered from the day of transplantation until day 100 after aHSCT for all recipients except for CMVseronegative recipients who received transplantation from CMV-seronegative donors. LET was administered per os as primary prophylaxis at $480 \mathrm{mg} /$ day or $240 \mathrm{mg} /$ day for patients concomitantly treated with cyclosporine. LET administration was discontinued on the development of csCMV infection.

\section{Statistical analysis}

The chi-square test or Fisher's exact test was used to compare categorical variables between groups of patients. For continuous variables, the Mann-Whitney $U$ test was used. The cumulative incidence of csCMV infection was evaluated using Gray's test ${ }^{38,39}$, with all-cause mortality and relapse as competing risks. The Kaplan-Meier method was used to estimate the probabilities of OS, and the log-rank test was used to compare OS between the two groups. Considering disease mortality and NRM as competing risks, the cumulative incidence of disease mortality and NRM were compared using Gray's test. The cumulative incidence of neutrophil/platelet/reticulocyte engraftment was also evaluated using Gray's test. Statistical significance was set at $P<0.05$. All statistical analyses were performed using EZR (Saitama Medical Center, Jichi Medical University; www. jichi.ac.jp/saitama-sct/SaitamaHP.files/statmedEN.html), a graphical user interface for R (The R Foundation for Statistical Computing, version 3.4.1, Vienna, Austria $)^{40}$.

\section{Declarations}

\section{Funding}

This work was supported by the First Department of Internal Medicine, Kansai Medical University, Osaka, Japan.

\section{Availability of data}

The original data for this study are available from the corresponding author on reasonable request.

\section{Code availability}

Not applicable

\section{Ethics approval}

This study was conducted with a waiver of informed consent following approval by the Research Ethics Committee of the Faculty of Medicine, Kansai Medical University.

\section{Acknowledgments}


The authors acknowledge all the doctors and medical professionals who supported our clinical practice. We would also like to thank Editage (www.editage.com) for English language editing.

\section{Author Contributions}

A.S wrote the main manuscript text and H.Y and A.S analyzed data. All authors reviewed the manuscript.

\section{Competing Interests}

The authors declare no competing interests.

\section{References}

1. Chen, K., Cheng, M. P., Hammond, S. P., Einsele, H. \& Marty, F. M. Antiviral prophylaxis for cytomegalovirus infection in allogeneic hematopoietic cell transplantation. Blood Adv.2, 2159-2175; 10.1182/bloodadvances.2018016493 (2018).

2. de la Cámara, R. CMV in Hematopoietic Stem Cell Transplantation. Mediterr. J. Hematol. Infect. Dis.8, e2016031; 10.4084/MJHID.2016.031 (2016).

3. Takenaka, K. et al. Cytomegalovirus reactivation after allogeneic hematopoietic stem cell transplantation is associated with a reduced risk of relapse in patients with acute myeloid leukemia who survived to Day 100 after transplantation: the Japan Society for Hematopoietic Cell Transplantation Transplantation-related complication working group. Biol. Blood Marrow Transplant.21, 2008-2016; 10.1016/j.bbmt.2015.07.019 (2015).

4. Teira, P. et al. Early cytomegalovirus reactivation remains associated with increased transplantrelated mortality in the current era: a CIBMTR analysis. Blood. 127, 2427-2438; 10.1182/blood-201511-679639 (2016).

5. Cantoni, N. et al. Evidence for a bidirectional relationship between cytomegalovirus replication and acute graft-versus-host disease. Biol. BloodMarrow Transplant.16, 1309-1314;

10.1016/j.bbmt.2010.03.020 (2010).

6. Gratwohl, A. et al. European Group for Blood and Marrow Transplantation and the European Leukemia Net. Risk score for outcome after allogeneic hematopoietic stem cell transplantation: a retrospective analysis. Cancer. 115, 4715-4726; 10.1002/cncr.24531 (2009).

7. Nichols, W. G., Corey, L., Gooley, T., Davis, C., \& Boeckh, M. High risk of death due to bacterial and fungal infection among cytomegalovirus (CMV)-seronegative recipients of stem cell transplants from seropositive donors: evidence for indirect effects of primary CMV infection. J. Infect. Dis.185, 273$282 ; 10.1086 / 338624$ (2002).

8. Yong, M. K. et al. Cytomegalovirus reactivation is associated with increased risk of late-onset invasive fungal disease after allogeneic hematopoietic stem cell transplantation: A multicenter study in the current era of viral load monitoring. Biol. Blood Marrow Transplant.23, 1961-1967; 10.1016/j.bbmt.2017.07.025 (2017). 
9. Chemaly, R. F. et al. AIC246 Study Team. Letermovir for cytomegalovirus prophylaxis in hematopoietic-cell transplantation. N. Engl. J. Med.370, 1781-1789; 10.1056/NEJMoa1309533 (2014).

10. Marty, F. M. et al. Letermovir prophylaxis for cytomegalovirus in hematopoietic-cell transplantation. N. Engl. J. Med.377, 2433-2444; 10.1056/NEJMoa1706640 (2017).

11. Ljungman, P. et al. 2017 European Conference on Infections in Leukaemia group. Guidelines for the management of cytomegalovirus infection in patients with haematological malignancies and after stem cell transplantation from the 2017 European Conference on Infections in leukaemia (ECIL 7). Lancet Infect. Dis.19, e260-e272; 10.1016/S1473-3099(19)30107-0 (2019).

12. Bacigalupo, A. et al. Defining the intensity of conditioning regimens: working definitions. Biol. Blood Marrow Transplant.15, 1628-1633; 10.1016/j.bbmt.2009.07.004 (2009).

13. Sorror, M. L. et al. Hematopoietic cell transplantation (HCT)-specific comorbidity index: a new tool for risk assessment before allogeneic HCT. Blood.106, 2912-2919; 10.1182/blood-2005-05-2004 (2005).

14. Armand, P. et al. Validation and refinement of the Disease Risk Index for allogeneic stem cell transplantation. Blood.123, 3664-3671; 10.1182/blood-2014-01-552984 (2014).

15. Przepiorka, D. et al. Consensus Conference on Acute GVHD Grading. Bone Marrow Transplant.15, 825-828 (1995).

16. Ljungman, P., Griffiths, P., \& Paya, C. Definitions of cytomegalovirus infection and disease in transplant recipients. Clin. Infect. Dis.34, 1094-1097; 10.1086/339329 (2002).

17. Gooley, T.A., Leisenring, W., Crowley, J., \& Storer, B. E. Estimation of failure probabilities in the presence of competing risks: new representations of old estimators. Stat. Med.18, 695-706; 10.1002/(sici)1097-0258(19990330)18:6<695::aid-sim60>3.0.c0;2-o (1999).

18. Scrucca, L., Santucci, A., \& Aversa, F. Competing risk analysis using R: an easy guide for clinicians. Bone Marrow Transplant. 40, 381-387; 10.1038/sj.bmt.1705727 (2007).

19. Kanda, Y. Investigation of the freely available easy-to-use software 'EZR' for medical statistics. Bone Marrow Transplant.48, 452-458, DOI: 10.1038/bmt.2012.244 (2013).

20. Boeckh, M. \& Nichols, W. G. The impact of cytomegalovirus serostatus of donor and recipient before hematopoietic stem cell transplantation in the era of antiviral prophylaxis and preemptive therapy. Blood.103, 2003-2008; 10.1182/blood-2003-10-3616 (2004).

21. Kanda, Y. et al. A randomized controlled trial of plasma real-time PCR and antigenemia assay for monitoring CMV infection after unrelated BMT. Bone Marrow Transplant. 45, 1325-1332; 10.1038/bmt.2009.337 (2010).

22. Mikulska, M. et al. CMV infection after transplant from cord blood compared to other alternative donors: the importance of donor-negative CMV serostatus. Biol. Blood Marrow Transplant.18, 92-99; 10.1016/j.bbmt.2011.05.015 (2012).

23. Takami, A., Mochizuki, K., Asakura, H., Yamazaki, H., Okumura, H., Nakao, S. High incidence of cytomegalovirus reactivation in adult recipients of an unrelated cord blood transplant. 
Haematologica. 90, 1290-1292 (2005).

24. Castillo, N. et al. Early and long-term impaired T lymphocyte immune reconstitution after cord blood transplantation with antithymocyte globulin. Biol. Blood Marrow Transplant. 23, 491-497; 10.1016/j.bbmt.2016.11.014 (2017).

25. Servais, S., Hannon, M., Peffault de Latour, R., Socie, G. \& Beguin, Y. Reconstitution of adaptive immunity after umbilical cord blood transplantation: impact on infectious complications. Stem Cell Investig. 4, 40; 10.21037/sci.2017.05.03 (2017).

26. Albano, M. S. et al. Umbilical cord blood transplantation and cytomegalovirus: posttransplantation infection and donor screening. Blood.108, 4275-4282; 10.1182/blood-2006-04-020313 (2006).

27. Yokoyama, H. et al. Effects of HLA mismatch on cytomegalovirus reactivation in cord blood transplantation. Bone Marrow Transplant.54, 1004-1012; 10.1038/s41409-018-0369-0 (2019).

28. Crocchiolo, R. et al. Infections after T-replete haploidentical transplantation and high-dose cyclophosphamide as graft-versus-host disease prophylaxis. Transpl. Infect. Dis.17, 242-249; 10.1111/tid.12365 (2015).

29. Goldsmith, S. R. et al. Cytomegalovirus viremia, disease, and impact on relapse in T-cell replete peripheral blood haploidentical hematopoietic cell transplantation with post-transplant cyclophosphamide. Haematologica.101, e465-e468; 10.3324/haematol.2016.149880 (2016).

30. Goldsmith, S. R. et al. Posttransplant cyclophosphamide is associated with increased cytomegalovirus infection: a CIBMTR analysis. Blood.137, 3291-3305; 10.1182/blood.2020009362 (2021).

31. Terao, T. et al. Letermovir administration to prevent cytomegalovirus reactivation is the potential risk of chronic graft-versus-host disease in patients who received haploidentical stem-cell transplantation with post-transplant cyclophosphamide. Front. Oncol. 11, 666774; 10.3389/fonc.2021.666774 (2021).

32. Green, M. L. et al. Cytomegalovirus viral load and mortality after haemopoietic stem cell transplantation in the era of pre-emptive therapy: a retrospective cohort study. Lancet Haematol. $\mathbf{3}$, e119-e127; 10.1016/S2352-3026(15)00289-6 (2016).

33. Duke, E. R. et al. CMV viral load kinetics as surrogate endpoints after allogeneic transplantation. J. Clin. Invest.131, e133960; 10.1172/JCl133960 (2021).

34. Ljungman, P. et al. A mortality analysis of Letermovir prophylaxis for cytomegalovirus (CMV) in CMVseropositive recipients of allogeneic hematopoietic cell transplantation. Clin. Infect. Dis.70, 15251533; 10.1093/cid/ciz490 (2020).

35. Sawayama, Y. et al. ATL Working Group of the Japan Society for Hematopoietic Cell Transplantation (2019) Cytomegalovirus reactivation is associated with increased mortality more than 100 days after allogeneic hematopoietic stem cell transplantation for adult T-cell leukemia/lymphoma. Am. J. Hematol.94, E143-E146; 10.1002/ajh.25438 (2019).

36. Mori, Y. et al. Efficacy of prophylactic letermovir for cytomegalovirus reactivation in hematopoietic cell transplantation: a multicenter real-world data. Bone Marrow Transplant. 56, 853-862; 
10.1038/s41409-020-01082-z (2021).

37. Marty, F. M. et al. Outcomes of patients with detectable CMV DNA at randomization in the phase III trial of letermovir for the prevention of CMV infection in allogeneic hematopoietic cell transplantation. Am. J. Transplant.20, 1703-1711; 10.1111/ajt.15764 (2020).

38. Solano, C. et al. Pre-engraftment cytomegalovirus DNAemia in allogeneic hematopoietic stem cell transplant recipients: incidence, risk factors, and clinical outcomes. Bone Marrow

Transplant. 54, 90-98; 10.1038/s41409-018-0251-0 (2019).

39. Derigs, P. et al. Letermovir prophylaxis is effective in preventing cytomegalovirus reactivation after allogeneic hematopoietic cell transplantation: single-center real-world data. Ann. Hematol.100, 2087-2093; 10.1007/s00277-020-04362-2 (2021).

40. Gooley T. A. et al. Reduced mortality after allogeneic hematopoietic-cell transplantation. N. Engl. J. Med. 363, 2091-2101; 10.1056/NEJMoa1004383 (2010).

\section{Figures}
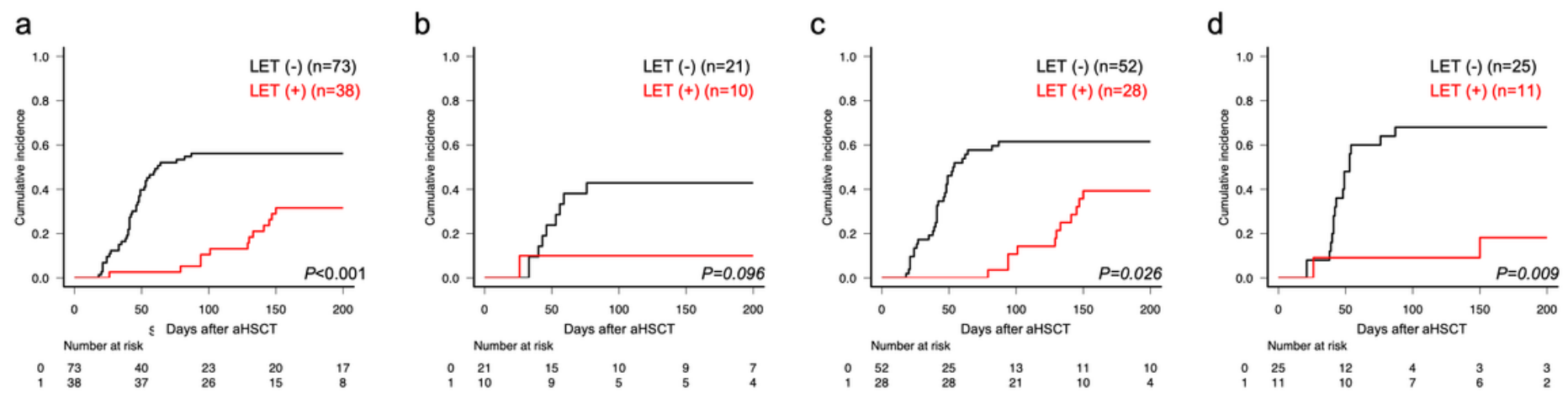

\section{Figure 1}

Cumulative incidence $(\mathrm{Cl})$ of clinically significant cytomegalovirus (csCMV) infection, in relation to letermovir prophylaxis in a time-to-event analysis. (a) $\mathrm{Cl}$ of csCMV infection in all patients $(n=111)$, (b) patients at low risk for CMV reactivation $(n=31)$, (c) patients at high risk for CMV reactivation $(n=80)$, and $(d)$ patients $\geq 55$ years $(n=36)$ 
a

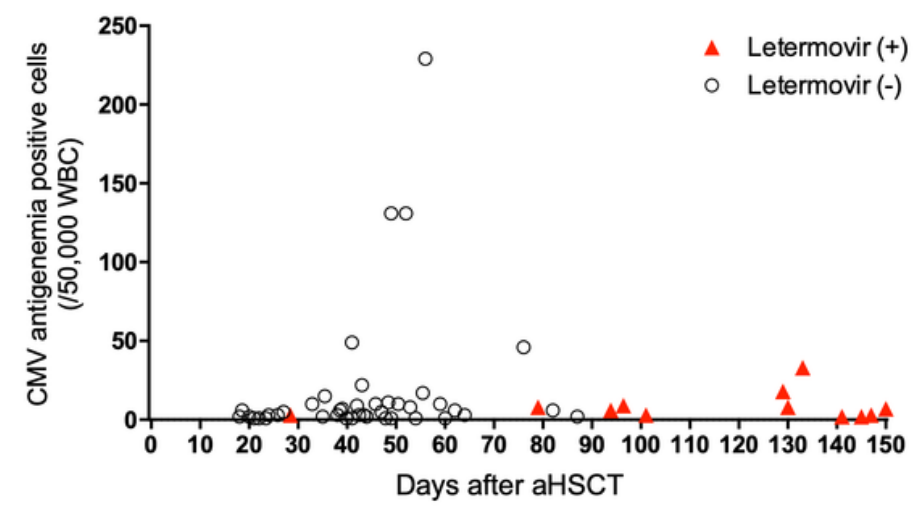

b

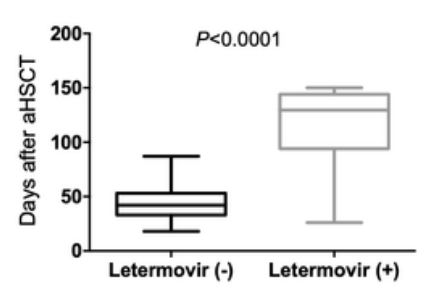

C

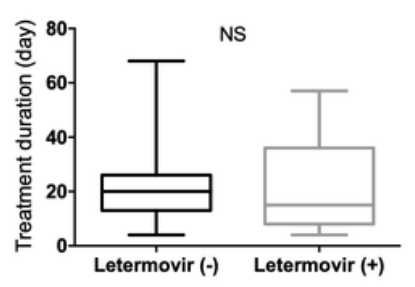

\section{Figure 2}

(a) Relationship between the number of CMV antigenemia-positive cells and the time from allogeneic hematopoietic stem cell transplantation (aHSCT) to initial preemptive therapy, (b) Start day from aHSCT to initial preemptive therapy, and (c) duration of initial preemptive therapy of patients with and without letermovir prophylaxis NS, not significant 
a

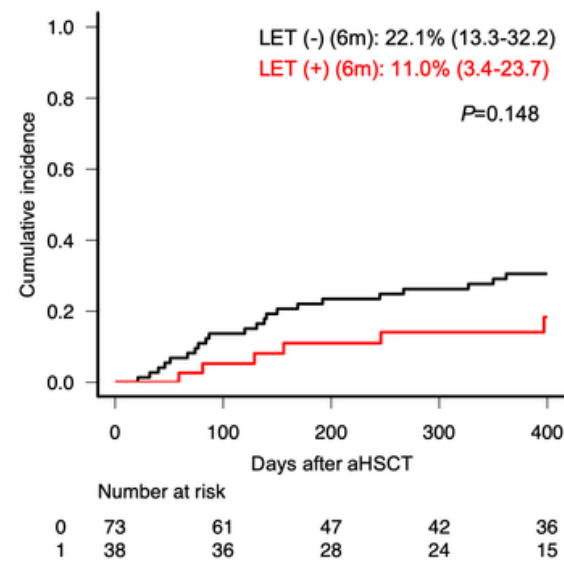

b

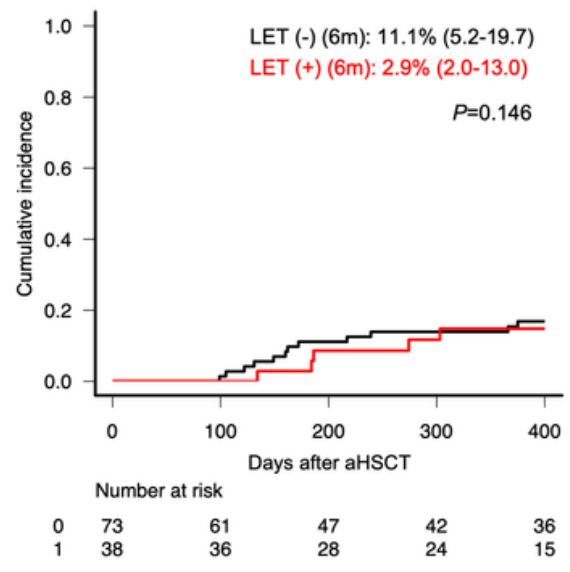

C

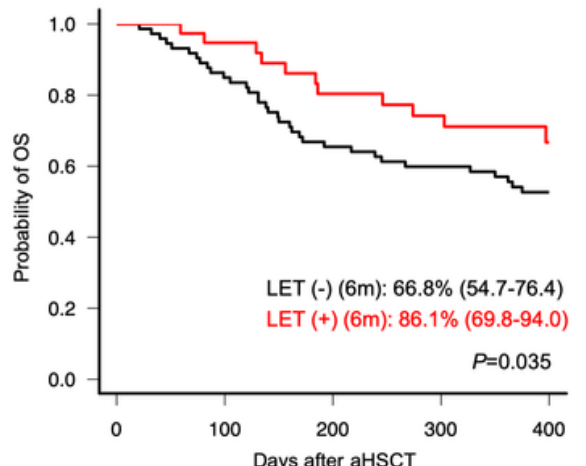

Number at risk

$\begin{array}{lllll}73 & 61 & 47 & 42 & 36 \\ 38 & 36 & 28 & 24 & 15\end{array}$

\section{Figure 3}

Cumulative incidence of (a) non-relapse mortality, (b) disease mortality, and (c) overall survival (OS) after allogeneic hematopoietic stem cell transplantation in patients with and without letermovir prophylaxis

a

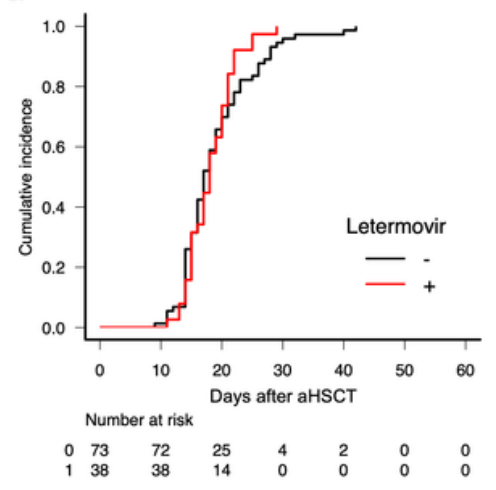

b

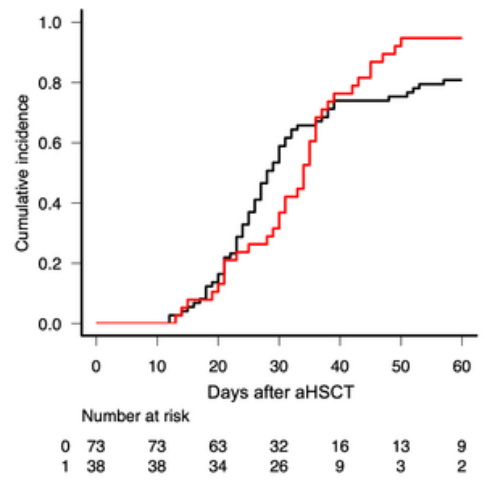

C

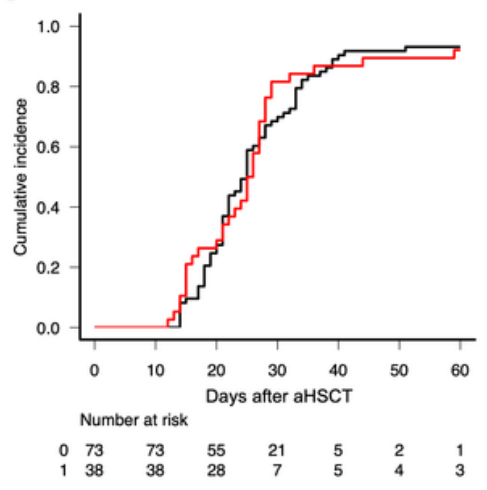




\section{Figure 4}

Cumulative incidence of (a) neutrophil engraftment, (b) platelets $(20 \times 109 / \mathrm{L})$ and $(\mathrm{c})$ reticulocytes $(1.0 \%)$ in patients with and without letermovir prophylaxis NS, not significant 